\title{
Estefanofilariose em bovinos
}

\author{
Stephanofilariasis in cattle
}

\section{Vanessa Issuzu Miyakawa ${ }^{1}$; Antonio Carlos Faria dos Reis²; Júlio Augusto Naylor Lisbôa ${ }^{3 *}$}

\section{Resumo}

A estefanofilariose é uma doença mundialmente distribuída e caracteriza-se por lesões na pele causadas por nematódeo do gênero Stephanofilaria. É mais prevalente no verão, devido à maior proliferação das moscas consideradas vetores do parasita, e acomete várias espécies animais. Nos bovinos caracterizase por uma dermatite crônica associada com erupção papular progredindo para nódulos, alopecia e ulceração crostosa. Apesar de ser uma doença conhecida há muitos anos, há poucos estudos e relatos sobre a mesma. A literatura é particularmente escassa no Brasil. Aspectos gerais sobre a epidemiologia, o agente, as manifestações e os métodos de diagnóstico, tratamento e prevenção foram revisados.

Palavras-chave: Stephanofilaria sp., bovinos, diagnóstico, tratamento

\begin{abstract}
Stephanofilariasis is a worldwide disease caused by the nematode Stephanofilaria that determines skin lesions in several definitive hosts. The prevalence is higher in summer due to the increase in fly population, the intermediate host. In cattle, the chronic dermatitis is characteristic begining with papules that progress to nodules, alopecia and ulcers with crusts. Despite it's long time recognition, there are few studies and reports about this disease, specially in Brazil. General informations of epidemiology, the agent, clinical signs, diagnosis methods, treatment and prevention were rewied.
\end{abstract}

Key words: Stephanofilaria sp., cattle, diagnosis, treatment

Médica Veterinária Mestre em Ciência Animal pela Universidade Estadual de Londrina. E-mail: issuzu@gmail.com

2 Docente do Departamento de Medicina Veterinária Preventiva da Universidade Estadual de Londrina. E-mail: reis@uel.br

3 Docente do Departamento de Clínicas Veterinárias da Universidade Estadual de Londrina; Campus Universitário, Londrina, PR, CEP. 86051-990. E-mail: janlisboa@uel.br

* Autor para correspondência 


\section{Introdução}

A estefanofilariose é uma dermatite crônica causada por nematódeo do gênero Stephanofilaria, mais prevalente no verão e transmitida por moscas. Está mundialmente distribuída e acomete várias espécies animais. Apesar de reconhecida há quase 80 anos, a literatura específica não é extensa, o que demonstra que a doença nunca foi alvo de grande interesse.

Existem poucas informações sobre epidemiologia, métodos diagnósticos e tratamento da doença no Brasil. As lesões, em bovinos, foram observadas na cabeça, na escápula, no teto, no jarrete (OBA et al., 1977), próximas à cauda, na garupa, na coxa (NOVAES et al., 1988), na quartela (NOVAES; OLIVEIRA; MOREIRA, 1990) e no úbere (BIRGEL et al., 1972; GAVA et al., 2006; MIYAKAWA; REIS; LISBÔA, 2007).

O diagnóstico presuntivo, baseado na história e na característica da lesão cutânea, é comumente confirmado pela resposta ao tratamento. A demonstração do nematódeo associado à lesão é, na maioria das vezes, frustrada em exames histopatológicos (GAVA et al., 2006), o que dificulta a confirmação do diagnóstico.

Algumas alternativas de tratamentos já foram apontadas como provavelmente eficientes. Destacam-se os organofosforados como o triclorfon e o coumafós para uso tópico (PATNAIK, 1970); a ivermectina por via parenteral (GILL et al., 1991); o levamisol por via parenteral (RAI et al., 1994); e o triclorfon tópico associado à ivermectina pour-on (MIYAKAWA; REIS; LISBÔA, 2006). O tempo para a recuperação completa da lesão varia de acordo com o tamanho original da mesma e comumente ultrapassa os 30 dias. Essa revisão tem como objetivo apresentar informações gerais e atualizadas sobre a estefanofilariose em bovinos.

\section{Aspectos epidemiológicos}

A estefanofilariose é uma doença mundialmente distribuída. Caracteriza-se por lesões na pele causadas pelo nematódeo do gênero Stephanofilaria (LAPAGE, 1976). Recebe nomes populares como "Úlcera da lactação", "Chagas de verão", "Cascado", "Hump-sore", "Flywarts", "Kriansore" entre outros.

As lesões em bovinos na América do Norte se localizam na região abdominal ventral e desenvolvem-se também no canto medial do olho. Na Indonésia a dermatite conhecida como "cascado" é encontrada no pescoço, na sobreunha, na cernelha e ao redor dos olhos. $\mathrm{Na}$ Índia e na Rússia, as feridas, conhecidas como "humpsore", localizam-se principalmente nos membros e na região da escápula (McGAVIN; ZACHARY, 2007). No Japão, Ueno e Chibana (1977) relataram lesões nos tetos e na mufla. Outras localizações já foram descritas em bovinos como a pele da bolsa escrotal (WATRELOT-VIRIEUX; PIN, 2006), o espaço interdigital, o peito e a base da cauda (CHATTERJEE; CHAKRABARTI, 1983).

No Brasil, os primeiros casos foram descritos no estado de São Paulo por Birgel et al. (1972) e por Oba et al. (1977). Além da espécie bovina, no Brasil, foi relatado um caso de dermatite nodular ulcerativa na região abdominal causada por Stephanofilaria sp. em cão (NOVAES, 2005) e recentemente, identificouse Stephanofilaria sp. em lesões conhecidas como úlcera varicosa indolente nos membros inferiores de pessoas portadoras crônicas dessas feridas (NOVAES; MIYASHIDA, 2007).

As lesões em bovinos, no Brasil, foram observadas na cabeça, na região escapular, no teto e no jarrete (OBA et al., 1977); próximo à cauda, na garupa, na coxa (NOVAES et al., 1988) e na quartela (NOVAES; OLIVEIRA; MOREIRA, 1990). São muito freqüentes na pele da mama (GAVA et al., 2006), e particularmente na porção anterior do úbere de vacas leiteiras lactantes (BIRGEL et al., 1972; MIYAKAWA; REIS; LISBÔA, 2007).

A prevalência da doença é maior durante a estação das chuvas prolongando-se por algum tempo após 
esse período. O número de casos se reduz durante o inverno, permanecendo quiescente durante essa época (PAL; SINHA, 1968). Na Alemanha, as "feridas de verão" aparecem em maio e junho (2 a 4 semanas após os animais terem iniciado o pastoreio) e desaparecem em outubro e novembro com o início do frio (GRÜNDER, 2005). No Brasil, Miyakawa, Reis e Lisbôa (2007) observaram maior prevalência nos meses de dezembro a março. Essa sazonalidade está relacionada à proliferação do vetor do parasita. A Musca conducens e a Haematobia irritans são apontados como hospedeiros intermediários e transmissores da Stephanofilaria spp para os bovinos (RADOSTITS; BLOOD; GAY, 1994). Um estudo em Queensland demonstrou que a distribuição da estefanofilariose nos bovinos coincide com a atividade da mosca Hematobia irritans exigua, sugerindo que essa mosca seja o vetor da doença na Austrália (JOHNSON; ARTHUR; SHEPHERD, 1986).

Ainda na Austrália, Johnson e Toleman (1988) investigaram casos naturais da doença durante quatro anos consecutivos e observaram que os zebuínos são mais resistentes do que os taurinos a essa parasitose.

\section{Agente etiológico e ciclo biológico}

$\mathrm{O}$ agente pertence ao filo Nemathelminthes, classe Nematoda, ordem Filarioidea, família Filariidae e gênero Stephanofilaria (LAPAGE, 1976). Existem onze espécies de Stephanofilaria identificadas em diferentes países. Entretanto, devido às semelhanças morfológicas, é provável que uma mesma espécie tenha recebido nomes distintos em cada país em que foi descrita (BAIN; VAN DER LUGT; KAZADI, 1996). Duas espécies não estão devidamente diferenciadas: a $S$. andamani encontrada em búfalos, nas Ilhas Andamã, e a $S$. srivastavi, observada em elefantes na Índia, podem ser a mesma espécie (BAIN; VAN DER LUGT; KAZADI, 1996). Segundo esses mesmos autores, quatro espécies são, provavelmente, sinônimas: a
S. dedoesi descrita em bovinos, caprinos e búfalos na Indonésia, em 1933, e as outras três observadas em bovinos; S. okinawaensis no Japão (UENO; CHIBANA, 1977), S. assamensis na Índia (PANDE, 1936) e S. kaeli na Malásia (BUCKLEY, 1937), essa última observada também em caprinos.

As outras espécies aceitas são a $S$. stilesi em bovinos nos EUA e na Rússia, a $S$. zaheeri em búfalos no Pasquitão, a $S$. dinniki em rinocerontes no Kenia e a $S$. thelazioides em hipopótamos no Sul da África (BOOMKER et al., 1995). A última espécie descrita foi a $S$. boomkeri em suínos no Zaire (BAIN; VAN DER LUGT; KAZADI, 1996). Segundo López-Neyra (1956) quatro espécies deviam ser consideradas: $S$. dedoesi, $S$. stilesi, $S$. assamensis e $S$. kaeli. Mais recentemente, Anderson (2000) indica a existência das seguintes espécies: $S$. stilesi, S. assamensis, S. kaeli e S. zaheeri. Nenhum dos poucos estudos realizados no Brasil identificou a espécie envolvida.

O gênero Stephanofilaria possui boca elevada com um anel de pequenos espinhos na periferia e, um pouco atrás destes, outro círculo de espinhos interrompidos por cerca de 4 a 5 grandes espinhos subdorsais. Existem aproximadamente 8 papilas no círculo externo. O esôfago é curto e não dividido. A cauda do macho é curta com numerosas papilas (LEVINE, 1980). As diferenças morfológicas entre as espécies relacionam-se, principalmente, ao número de espinhos labiais (interno e externo), papilas cefálicas, tamanho das microfilárias, características da vulva, vagina, cauda e corpo (BOOMKER et al., 1995). O comprimento do macho adulto pode variar entre 2 e $4 \mathrm{~mm}$, e o da fêmea entre 5 e $11 \mathrm{~mm}$. As microfilárias medem de 18 a $52 \mu \mathrm{m}$ (LEVINE, 1980).

Patnaik (1973) demonstrou que as microfilárias da $S$. assamensis ingeridas pela Musca conducens permanecem de 23 a 25 dias em diferentes localizações no organismo desse hospedeiro intermediário, até atingirem o estágio infectante. Durante esse período realizam duas ecdises, uma entre 5 e 6 dias e outra após 13 a 15 dias. As 
larvas com capacidade infectante localizam-se na probóscide do inseto facilitando a transmissão para o hospedeiro definitivo. O período pré-patente na pele do bovino pode variar de 3 a 8 semanas (HIBLER, 1966) até 72 dias (PATNAIK, 1973).

Após a deposição na pele do hospedeiro definitivo, o tempo necessário para as larvas atingirem a maturidade é incerto. Os adultos vivem na base dos folículos pilosos, as fêmeas são vivíparas e as microfilárias apresentam-se livres mais profundamente na derme ou mais superficialmente no exsudato das lesões cutâneas (MAXIE, 2007), ocasião em que são ingeridas pelas moscas atraídas.

Novaes e Miyashida (2007) observaram microfilárias no sangue periférico e na pele de pessoas não portadoras de feridas e no cordão umbilical e sangue da circulação periférica colhido no momento da realização do "teste do pezinho", de bebês recém-nascidos de mães não portadoras de úlceras cutâneas. Esse fato aponta a possibilidade da transmissão transplacentária em humanos.

\section{Sinais clínicos e diagnóstico}

Os sinais clínicos da estefanofilariose caracterizam-se inicialmente por uma dermatite, com erupção papular progredindo para nódulos, alopecia e úlcera crostosa (WHITE; EVANS, 2002, MIYAKAWA; REIS; LISBÔA, 2006) (Figura 1). As feridas possuem, comumente, formato circular. Feridas iniciais geralmente medem $1 \mathrm{~cm}$ de diâmetro e com o tempo de evolução maior podem atingir aproximadamente $25 \mathrm{~cm}$ de diâmetro (MAXIE, 2007). O prurido e exsudato sero-sanguinolento estão presentes na maioria dos casos (SMITH, 1986). A lesão, única ou não, permanece por um longo tempo se não tratada, podendo persistir por dois a três anos (SCOTT, 1988; ROSSER JÚNIOR, 1993; WHITE; EVANS, 2002). A cura espontânea é possível após esse período tão longo. As feridas vão adquirindo aspecto seco e crostoso e regredindo em seu tamanho paulatinamente até a cicatrização.
É frequente, no local da lesão permanecer por um tempo uma cicatriz elevada e alopécica(GRÜNDER, 2005). As lesões atraem moscas o que predispõe ao desenvolvimento de mí́ases. A localização das lesões na proximidade do teto pode causar mastite (SMITH, 1981).

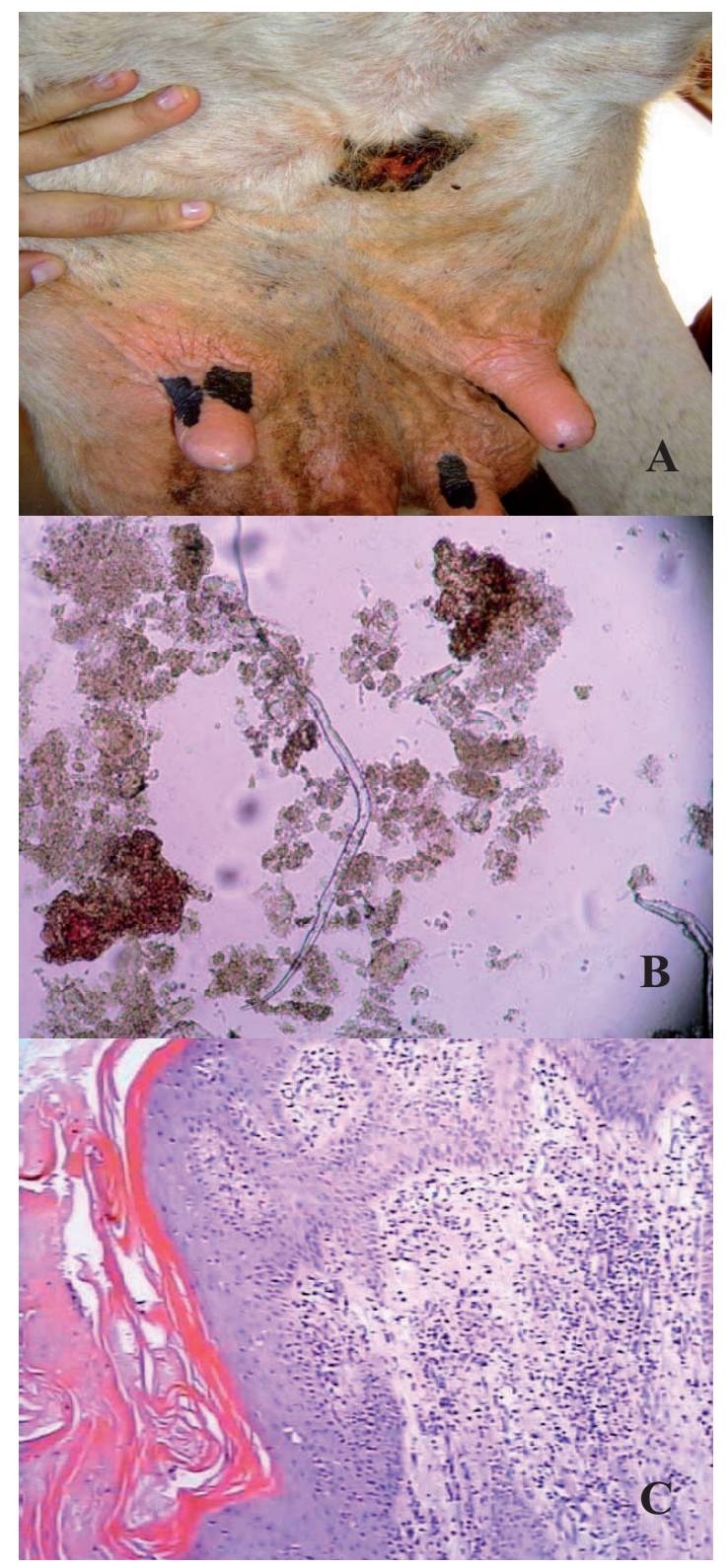

Figura 1. Aspectos clínicos e diagnóstico da estefanofilariose. A) ferida característica ulcerada com crostas e exsudato sero-sanguinolento, localizada próximo à linha média ventral em posição cranial do úbere de uma vaca leiteira; B) forma adulta de Stephanofilaria sp. no exame direto do sedimento; $\mathrm{C}$ ) exame histopatológico de tecido biopsado da lesão demonstrando a hiperqueratose ortoqueratótica acentuada e a reação inflamatória intensa de derme (HE 10x). 
O diagnóstico é baseado na história, no exame físico e na resposta ao tratamento. É confirmado somente por técnicas que revelem o parasita. Para esse fim, pode-se empregar o exame histopatológico de tecidos biopsados na proximidade da borda da lesão (ODUYE, 1971). Impressões com lâminas na ferida contendo exsudato ou raspado da borda da ferida seguido da confecção de esfregaços em lâmina podem ser corados com Giemsa ou vermelho Congo para a visualização das microfilárias (NOVAES, 2005). Ueno e Chibana (1977) descreveram um método de exame direto por microscopia que se baseia na migração dos parasitas contidos no interior do tecido removido cirurgicamente para a solução salina fisiológica $(\mathrm{NaCl} 0,9 \%)$ na qual o tecido fica embebido. $\mathrm{O}$ exame do sedimento após centrifugação permite a visualização das formas adultas e das microfilárias. A eficiência desse método para a confirmação da presença do parasita (Figura 1) foi comprovada em casos naturais da doença (MIYAKAWA; REIS; LISBÔA, 2007).

Os achados histopatológicos consistem em uma dermatite perivascular, superficial e profunda, associada com eosinofilia e infiltrado mononuclear, principalmente linfocitário (MAXIE, 2007; McGAVIN; ZACHARY, 2007) (Figura 1). Maxie (2007) e Gava et al. (2006) descreveram a presença de microabscessos contendo eosinófilos e células mononucleares na epiderme. Congestão, hemorragia, paraqueratose, acantose, reações granulomatosas na epiderme e glândulas sebáceas atrofiadas ou degeneradas são observações frequentes (DAS; TRIPATHY; MISRA, 1974; MAXIE, 2007; McGAVIN; ZACHARY, 2007).

O parasita adulto geralmente é observado na epiderme e na derme, próximo ao folículo piloso e às glândulas sebáceas em formações císticas. As microfilárias são encontradas principalmente na derme (DIES; PRITCHARD, 1985). Apesar da histopatologia ser o método mais indicado, na literatura, para a confirmação da presença do nematódeo (SCOTT, 1988; 2007; SMITH, 1981; 1986; ROSSER JÚNIOR, 1993; WHITE; EVANS,
2002), Gava et al. (2006) e Miyakawa, Reis e Lisbôa (2007) realizaram exame histopatológico de 35 e de 24 vacas acometidas, respectivamente, e não observaram nenhuma forma adulta e/ou larvar de Stephanofilaria spp.

As microfilárias nos primeiros estágios apresentam formato estreito semelhante a um "C", com cabeça romba e corpo cilíndrico terminando em uma cauda afilada (RAO et al., 1979), e devem ser diferenciadas das microfilárias de outros parasitas como Oncocerca e Setária (LOKE; RAMACHANDRAN, 1966).

Para o diagnóstico diferencial deve-se considerar principalmente o eczema de úbere (GRÜNDER, 2005; GEORGE et al., 2008). A dermatofitose, a dermatofilose, a dermatite de contato, a dermatite por picadas de inseto, a paraqueratose por deficiência de zinco e a sarna corióptica podem ser também relacionadas (SCOTT, 1988; 2007).

\section{Tratamento e prevenção}

Os resultados dos tratamentos são variáveis quanto à eficácia, tempo de duração e possibilidade de recidivas. Não há um produto comercial específico para essa finalidade (SMITH, 1981). Entre os tratamentos mais citados destacam-se a aplicação tópica de organofosforados como o coumafós $2 \% \mathrm{e}$ o triclorfon 6\% (TAYLOR; HUNTER; ANDREWS, 2004). O triclorfon $6 \%$ aplicado topicamente mostrou-se eficaz para a eliminação do parasita, tanto na sua forma adulta quanto na larvar, e não houve recidivas (SRIVASTAVA; MALVIYA, 1968). O levamisol por via parenteral (9 a 12mg/ kg PV) em dose única associado ao óxido de zinco com uso tópico, uma vez ao dia até a cicatrização da lesão, promoveu a cura de $100 \%$ das feridas leves e moderadas e $92,9 \%$ das graves (RAI et al., 1994). A ivermectina por via parenteral $(200 \mu \mathrm{g} / \mathrm{kg})$ em dose única reduziu o número de microfilárias das feridas na orelha de búfalos (GILL et al., 1991). A ivermectina em pasta (concentrações de $1 \%$ e de $2 \%$ ) foi aplicada topicamente em feridas provocadas 
por Stephanofilaria sp. no úbere de vacas e resultou na cicatrização de todos os casos (MIYAKAWA; REIS; LISBÔA, 2006).

O tempo para a recuperação completa da lesão varia de acordo com o tamanho original da mesma e comumente o tratamento ultrapassa os 30 dias. Gründer (2005), para evitar recidivas, recomenda o uso sistêmico de anti-helmínticos específicos contra as estefanofilárias, tais como: o levamisol $(7,5 \mathrm{mg}$ / $\mathrm{kg})$ ou a ivermectina $(200 \mu \mathrm{g} / \mathrm{kg})$. Como a doença é frequente em vacas lactantes (BIRGEL et al., 1972; MIYAKAWA; REIS; LISBÔA, 2007), as opções de tratamento sistêmico, ainda que eficientes, poderão ser utilizadas somente após o término da lactação, evitando-se o aparecimento de resíduos indesejáveis no leite.

Patnaik (1970) afirmou que uma infecção constante e repetida com microfilárias é necessária para a manutenção da lesão e que o tratamento de todos os casos com um anti-helmíntico apropriado tem efeito controle, pois, ao exterminar as larvas, impede que as moscas se infectem. Outra medida de controle recomendada é um programa efetivo de redução de moscas no ambiente (SMITH, 1986), principalmente nas situações de apresentação enzoótica da doença (GRÜNDER, 2005).

\section{Referências}

ANDERSON, R. C. Nematodes parasites of vertebrates - their development and transmission. New York: CABI Publishing, 2000. 154 p.

BAIN, O.; VAN DER LUGT, J.; KAZADI, L. M. Stephanofilaria boomkeri $\mathrm{n} . \mathrm{sp}$., as a cause of severe skin disease in pigs in Zaire. Parasite, Paris, v. 3, n. 4, p. 377 $381,1996$.

BIRGEL, E. H.; PEREIRA P. C.; MEIRELLES, C. O. S.; AMARAL, V.; ARAÚJO, L. M. Úlcera da lactação filariose provavelmente determinada por Stephanofilaria. Atualidades Veterinárias, São Paulo, v. 1, n. 2, p. 56, 1972.

BOOMKER, J.; BAIN, O.; CHABAUD, A.; KRIEK, N. P. J. Stephanofilaria thelazoides n.sp. (Nematoda: Filariidae) from a hippopotamus and its affinities with the species parasitic in the African black rhinoceros. Systematic Parasitology, Berlin, v. 32, n. 2, p. 205-210, 1995.

BUCKLEY, J. J. C. On a new species of Stephanofilaria causing lesions in the legs of cattle in the Malay Peninsula. Journal of Helminthology, Cambridge, v. 15, n. 4, p. 233242, 1937.

CHATTERJEE, A.; CHAKRABARTI, A. Some uncommon lesions of Stephanofilarial dermatitis in cattle of West Bengal (India). Indian Journal of Animal Health, Kolkata, v. 22, n. 2, p. 67-70, 1983.

DAS, P. K.; TRIPATHY, S. B.; MISRA, S. K. Studies on the pathoanatomy of the skin in Stephanofilaria assamensis Pande, 1936 infection in cattle. Journal of Animal Science, Savoy, v. 45, n. 8, p. 543-545, 1974.

DIES, K. H.; PRITCHARD, J. Bovine stephanofilarial dermatitis in alberta. Canadian Veterinary Journal, Ottawa, v. 26, n. 11, p. 361-362, 1985.

GAVA, A.; MEZAROBA, S.; LUCIOLI, J.; FURLAN, F. H.; TRAVERSO, S. D. Stephanofilariose em bovinos no Estado de Santa Catarina: aspectos clínicos e lesionais. In: SEMINÁRIO DE INICIAÇÃO CIENTÍFICA CIÊNCIAS AGRÁRIAS, 16., 2006, Lages. Resumos.... Lages: UDESC, 2006. p. 95.

GEORGE, L. W.; DIVERS, T. J.; DUCHARM, N.; WELCOME, F. L. Diseases of the teats and udder. In DIVERS, T. J.; PEEK, S. F. Diseases of dairy cattle. 2. ed. Missouri: Elsevier, 2008. p. 327-394.

GILL, B. S.; BALAKRISHNAM, P.; LUMSDEN, G. G.; JONES, P. G. H. Treatment of stephanofilariosis ("earsore") with ivermectin. Veterinary Parasitology, Amsterdam, v. 40, n. 1/2, p. 159-163, 1991.

GRÜNDER, H. D. Stefanofilariosis. In: DIRKSEN, G.; GRÜNDER, H. D.; STÖBER, M. Medicina interna y cirúrgia del bovino. 4. ed. Buenos Aires: Inter-Médica, 2005. p. 66-68. v. 1.

HIBLER, C. P. Development of Stephanofilaria stilesi in the horn fly. The Journal of Parasitology, Nebraska, v. 52 , n. 5, p. 890-898, 1966.

JOHNSON, S. J.; ARTHUR, R. J.; SHEPHERD, R. K. The distribution and prevalence of stephanofilariasis en cattle in Queensland. Australian Veterinary Journal, New South Wales, v. 63, n. 4, p. 121-124, 1986.

JOHNSON, S. J.; TOLEMAN, M.A. Prevalence of stephanofilariasis in young Bos indicus cattle in northern Australia. Veterinary parasitology, Amsterdam, v. 29, n. 4, p. 333-339, 1988.

LAPAGE, G. Parasitologia veterinária. 4. ed. México: Compañia Editorial Continental S.A., 1976. 
LEVINE, N. D. Nematode parasites of domestic animals and of man. 2. ed. Mineapolis: Burgess Publishing Company, 1980.

LOKE, Y. W.; RAMACHANDRAN, C. P. Histopathology of Stephanofilaria kaeli lesions in cattle. Medical Journal of Malaya, Jalan Pahang, v. 20, n. 4, p. 348, 1966.

LÓPEZ-NEYRA, C. R. Revision de la superfamilia filarioidea (Weinland, 1858). Revista Ibérica de Parasitologia, Granada, v. 16, n. 1/2, p. 3-232, 1956.

MAXIE, M. G. Pathology of domestic animals. 5. ed. Philadelphia: Saunders Elsevier, 2007.

McGAVIN, M. D.; ZACHARY, J. F. Pathologic basis of veterinary disease. 4. ed. Missouri: Mosby Elsevier, 2007.

MIYAKAWA, V. I.; REIS, A.C. F.; LISBÔA, J. A. N. Tratamento da estefanofilariose em vacas leiteiras resultados preliminares. In: CONBRAVET, 33., 2006, Cuiabá. Anais...Cuiabá: SBMV, 2006. 1 CD-ROM.

- Aspectos epidemiológicos e clínicos da estefanofilariose em vacas leiteiras. Archives of Veterinary Science, Curitiba, v. 12, p. 171-172, 2007. Suplemento.

NOVAES, A. P. Estefanofilariose e dermatite nodular ulcerativa em cão: relato de caso. Revista de Educação Continuada do Conselho Regional de Medicina Veterinária, São Paulo, v. 8, n. 2, p. 93-97, 2005.

NOVAES, A. P.; COSTA, A. J. S.; BARBOSA, R. T.; MOREIRA, D. P.; RUZZA, F. J. Dermatite ulcerosa em bovinos provocada por Stephanofilaria. Pesquisa Agropecuária Brasileira, Brasília, v. 3, n. 8, p. 927-929, 1988.

NOVAES, A. P.; MIYASHIDA, A. T. Estefanofilariose em humanos: ocorrência e mecanismos de transmissão. Revista da Sociedade Brasileira de Medicina Tropical, Rio de Janeiro, v. 40, n. 2, p. 250-252, 2007.

NOVAES, A. P.; OLIVEIRA, M. C. S.; MOREIRA, D. P. Stephanofilaria sp: associada a casos de pododermatite em bovinos leiteiros. Arquivos de Biologia e Tecnologia, Curitiba, v. 33, n. 3, p. 575-579, 1990.

OBA, M. S. P.; SINHORINI, I. L.; NOVAES, A. P.; COSTA, A. J. S.; PORTO A. D. Stephanofilaria em bovinos do município de São Carlos, Estado de São Paulo. In: CONFERÊNCIA ANUAL DA SOCIEDADE PAULISTA DE MEDICINA VETERINÁRIA, 32., 1977, Pirassununga. Anais... Pirassununga: SPMV, 1977. p. 12.

ODUYE, O. O. Stephanofilarial dermatitis of cattle in Nigeria. Journal of Comparative Pathology, Amsterdam, v. 81, n. 6 , p. 581-583, 1971.
PAL, A. K.; SINHA, P. K. Stephanofilaria assamensis as the cause of common chronic ulcerated growth at the base of the dewclaws in cattle in west Bengal. The Indian Veterinary Journal, Chennai, v. 48, n. 2, p. 190-193, 1968.

PANDE, P. G. On the identity of the nematode worm recovered from humpsore of cattle in India. Indian Journal Veterinary Science and Animal Husbandry, New Delhi, v. 6, n. 4, p. 346-351, 1936.

PATNAIK, B. Studies on stephanofilariasis in orissa: V. treatment and control of "humpsore" in cattle due to Stephanofilaria assamensis. Indian Journal Animal Science, New Delhi, v. 40, n. 2, p. 167-174, 1970.

Studies on stephanofilariasis in orissa. III.

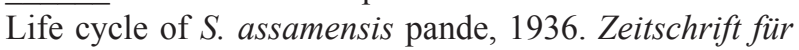
Tropenmedizin und Parasitologie, Stuttgart, v. 24, n. 6, p. $457-466,1973$.

RADOSTITS, O. M.; BLOOD, D. C.; GAY, C. C. Veterinary medicine. 8. ed. London: Baillière Tindall, 1994.

RAI, R. B.; AHLAWAT, S. P. S.; SINGH, S.; NAGARAJAN, V. Levamisole hydrochloride: an effective treatment for stephanofilarial dermatitis (Humpsore) in cattle. Tropical Animal Health and Production, Berlin, v. 26, n. 3, p.175-176, 1994.

RAO, B.V.; RAMAKRISHNA REDDY, P.; SUBBA REDDY, K.; PATNAIK, B. On the occurrence of Stephanofilaria assamensis var: bubalensis in Cuddapah district: Andhra Pradesh. The Indian Veterinary Journal, Chennai, v. 56, n. 5, p. 250-251, 1979.

ROSSER JÚNIOR, E. J. Parasitic dermatoses. In: HOWARD, J. L. Current veterinary therapy: food animal practice. 3. ed. Philadelphia: W.B. Saunders Company, 1993. p. 882-890.

SCOTT, D.W. Large animal dermatology. Philadelphia: W.B. Saunders Company, 1988.

. Color atlas of farm animal dermatology. Ames: Blackwell Publishing, 2007.

SMITH, J. P. Fly infestions. In: HOWARD, J. L. Current veterinary therapy: food animal practice. Philadelphia: W.B. Saunders Company, 1981. p. 1148-1152.

Fly infestions. In: HOWARD, J. L. Current veterinary therapy: food animal practice. 2. ed. Philadelphia: W.B. Saunders Company, 1986. p. 913916.

SRIVASTAVA, H. D.; MALVIYA, H. C. Treatment of "humpsore" in cattle caused by Stephanofilaria assamensis. The Indian Veterinary Journal, Chennai, v. 45, n. 8, p. 484-488, 1968. 
TAYLOR, S. M.; HUNTER, A. G.; ANDREWS, A. H. WATRElOT-VIRIEUX, D.; PIN, D. Chronic Ectoparasites, tick and arthropod-borne diseases. In: eosinophilic dermatitis in the scrotal area associated with ANDREWS, A. H.; BLOWEY, R. N.; BOYD, H.; EDDY stephanofilariasis infestation of charolais bull in France. R. G. Bovine medicine diseases and husbandry of cattle. Journal of Veterinary Medicine, Oxford, v. 53, n. 3, p. 2. ed. Ames: Blackwell Publishing, 2004. p. 740-777.

UENO, H.; CHIBANA, T. Stephanofilaria okinawaensis n. sp. from cutaneous lesions on the teats of cows in Japan. National of Animal Health Quarterly, Tokyo, v. 17, n. 1, p. 16-26, 1977. 150-152, 2006.

WHITE, S. D.; EVANS, A. G. Parasitic skin diseases. In: SMITH, B. P. Large animal internal medicine. 3. ed. St. Louis: Mosby, 2002. p. 1215-1222. 